\title{
Comparison of the Prevalences of Demodex folliculorum in Rosacea and Seborrhoeic Dermatitis with Standardized Skin Surface Biopsy
}

\author{
B Tas ${ }^{1}$, B Ozdemir², M Ilktac², B Taskin ${ }^{3}, \mathrm{EM} \mathrm{Savk}^{1}, \mathrm{C}^{2}$ Ozdalgicoglu²
}

\begin{abstract}
Objective: Rosacea (RSC) and seborrhoeic dermatitis (SD) are inflammatory dermatological conditions which are located in sebaceous gland-rich areas. Demodex folliculorum (D folliculorum) is the most common ectoparasite in humans that may play a role in the aetiopathogenesis of both diseases. The aims of this study were to compare the prevalences of $\mathrm{D}$ folliculorum positivity in patients with RSC and $S D$; compare the positivity in both diseases according to different age ranges and gender and compare mite positivity according to clinical types of RSC.

Methods: A total of 200 facial skin biopsies, one from each patient clinically diagnosed as RSC (100 patients) and as SD (100 patients) were studied between February 2011 and August 2013. D folliculorum density was investigated using standardized skin surface biopsy and the specimens were examined under light microscope. Quantitative data were tested with Pearson's Chi-squared test and Fisher's exact test, and significance was defined as a p-value of $<0.05$.

Results: Demodex mite was positive in 51 (25.50\%) of the 200 patients $(74.51 \%$ of them had RSC, and $25.49 \%$ had SD; $\mathrm{p}=0.0001)$. No statistical differences were detected between the prevalences of Demodex positivity in patients with RSC in terms of gender $(\mathrm{p}=0.768)$ and age $(\mathrm{p}=0.193$. In the $S D$ group, there was no statistical difference in mite positivity in terms of gender $(\mathrm{p}=0.138)$, while the positivity was significantly higher in the age group 45 years and over $(\mathrm{p}=0.039)$. In different clinical types of RSC, there was no significant difference with respect to mite positivity $(\mathrm{p}=0.168)$.

Conclusion: Positivity of D folliculorum was significantly higher in RSC when compared to SD. Our results suggest that $\mathrm{D}$ folliculorum mite can play a more important role in the aetiopathogenesis of RSC than $S D$.
\end{abstract}

Keywords: Demodex folliculorum, ectoparasite, rosacea, seborrhoeic dermatitis, standardized skin surfaced biopsy

WIMJ Open 2015; 2 (3): 127

\section{INTRODUCTION}

Rosacea (RSC) is a common chronic inflammatory disease of the face and occurs in approximately $3 \%$ of the population (1, 2 ). The lesions of the disease are mostly located in the central part of the face, especially the checks, nose and chin $(2,3)$. The disease is usually classified as erythematotelangiectatic, papulopustular, phymatous and ocular RSC. It is usually diagnosed with clinical features such as flushing erythema, permanent erythema and/or telangiectasias on the convexities of the face. The findings that help in the

From: ${ }^{1}$ Department of Dermatology, ${ }^{2}$ Department of Microbiology, Bagcilar Research and Training Hospital, Istanbul, Turkey and ${ }^{3}$ Department of Dermatology, Istanbul Bilim University, Istanbul, Turkey.

Correspondence: Dr B Tas, Atakoy 7-8, Kisim, Marti Sitesi, Marti 14/105, Bakirkoy, Istanbul, Turkey. Fax: 0-212-4404000; e-mail: betulavc@yahoo. com diagnosis are feelings of burning, tingling and dryness of the skin, oedema, ocular symptoms and/or hyperplastic changes $(2,4)$. Another inflammatory skin disease usually located in the middle of the face is seborrhoeic dermatitis (SD). The prevalence of the disease is $1-3 \%$. It is diagnosed by erythematous, thin, oily and yellow flakes on the face, scalp, chest, back and flexural areas which are rich in sebaceous glands (3-5).

Demodex mites ( $D$ folliculorum and $D$ brevis), which belong to the family of Demodicidae, are found on human skin as asymptomatic, saprophytic, permanent ectoparasites (6-8). D folliculorum has been found to be the most common ectoparasite in humans $(6,9,10)$, and occurs in $10 \%$ of skin biopsies and $12 \%$ of follicles $(9,11,12)$. It can play a pathogenetic role in various dermatological conditions $(6,7$, 13, 14). 


\section{SUBJECTS AND METHODS}

In this analytical, cross-sectional and comparative study, a total of 200 patients older than 18 years of both genders (100 with RSC and 100 with SD; mean age: 42.175 years), who were admitted to the outpatient clinic of the dermatology department of our hospital, were studied between February 2011 and August 2013. The study was approved by the ethics committee of our hospital (approval number: KAEK-201316/135). Written informed consent was obtained from each patient for a skin surface biopsy. The findings such as erythematotelangiectatic patches, papulopustules on an erythematous and/or oedematous ground, and hyperplastic skin changes as rhinophyma were considered as the diagnosis of the patients with RSC. Patients with ocular RSC were not included in the study. For the patients with SD, observational findings such as yellowish-pink, erythematous patches or plaques covered with thin, yellow, adherent, dry or oily squamae localized on the face, and symptoms such as pruritus and burning sensation were considered as clinically diagnostic. The skin biopsies of the lesions for each patient were taken from one of five different areas which were considered to be most intense and prominent. These areas were classified as forehead and eyebrow, cheeks, nasolabial folds, nose, and chin. All of the patients were grouped according to gender and age.

The age ranges were determined as $18-30,31-44$, and 45 years and over. Exclusion criteria of the patients were: age under 18 years; received systemic or local corticosteroid or immunosuppressive treatment in the previous month; presence of any immunosuppressive disease; and pregnant women. Mites were examined using standardized skin surface biopsy (SSSB). For the technique, a standard surface area of $1 \mathrm{~cm}^{2}$ was marked with a blue waterproof marker on the slide. Thereafter, one side of it was coated with cyanoacrilate adhesive and the adhesive side of the slide was pressed onto the lesion for 60 seconds then peeled off. After the slide was removed from the skin, it was covered with glycerine and examined under a light microscope (with $\times 40$ and $\times 100$ magnifications). In the examination of all the slides, the mites which were $0.3-0.4 \mathrm{~mm}$ long, and had four pairs of legs on the front part of the body were noted. Mite density was calculated as the number of mites per marked square centimetre. In the evaluation, a mite count of $\geq 5 / \mathrm{cm}^{2}$ area was considered an infestation. The Demodex counts and the mean density were determined with regard to the mite- positive patients in both diseases. The evaluations of the study were based on comparative analyses of clinical and laboratory data. The results were compared in terms of the following aspects: (i) distribution of the patients, according to gender and different age ranges in both disease groups; (ii) mite positivity according to different age ranges and gender in both diseases; (iii) mite positivity according to different clinical types of RSC and (iv) the difference in mite positivity between RSC and SD patients. Comparisons of quantitative data were tested with Pearson's Chi-squared and Fisher's exact tests. Significance of the results was evaluated according to $a p$-value of $<0.05$.

\section{RESULTS}

One hundred patients with RSC (72 women, 28 men) whose age range was 19-82 (mean age 51.13) years and 100 patients with SD (58 women, 42 men) whose age range was 18-64 (mean age 38.15) years were enrolled in the study. In the RSC group, the number of patients in the 18-30-year age range was eight (seven women, one man), in the 31-44-year age range, it was 30 ( 29 women, one man), and in the 45 years and over group, it was 62 ( 36 women, 26 men). The prevalence of RSC in the 18-30 and 31-44-year age ranges of women was significantly higher than in the men of their respective age groups $(p=0.003)$. In the $\mathrm{SD}$ group, the distribution of the patients were 48 ( 29 women, 19 men), 35 (20 women, $15 \mathrm{men}$ ) and 17 (nine women, eight men), respectively in the 18-30, 31-44 and $\geq 45$-year age ranges. The incidence of SD in the two genders in all age groups did not significantly differ from each other $(p=0.859)$.

In the RSC group, distribution of the mite-positive areas which had the most prominent lesions was the forehead in three (one woman, two men), the cheeks, 17 (13 women, four men), the chin, five (five women) and the nose, 13 (nine women, four men); there was none in the nasolabial sulcus. In the SD group, the distributions were the forehead in eight (six women, two men), the cheeks, three (three women), the nasolabial sulcus, one (one woman), the nose, one (one man) and the chin in zero. The minimum and maximum mite counts were 4 and 24 , respectively and the mean value was 8.78 in the RSC group, while in the SD group, these were 5 and 40 , and 11.15 , respectively. There were no statistically significant differences in the patients with RSC in terms of gender $(p=0.769)$ and age $(p=0.193)$ according to $D$ folliculorum positivity (Table 1).

Table 1: Distribution and prevalences of all the rosacea (RSC) cases in terms of gender and age according to $D$ folliculorum positivity

\begin{tabular}{llrlrrr}
\hline RSC group & & Demodex-negative & Demodex-positive & $\boldsymbol{p}$-values \\
\hline \multirow{2}{*}{ Age range (years) } & $18-30$ & 7 & $11.29 \%$ & 3 & $7.89 \%$ & \\
& $31-44$ & 14 & $22.58 \%$ & 15 & $39.47 \%$ & \\
\multirow{3}{*}{ Gender } & $\geq 45$ & 41 & $66.13 \%$ & 20 & $52.63 \%$ & 0.193 \\
& Male & 18 & $29.03 \%$ & 10 & $26.32 \%$ & \\
& Female & 44 & $70.97 \%$ & 28 & $73.68 \%$ & 0.769 \\
\hline
\end{tabular}


In the SD group, there was no statistical significance in terms of gender according to mite positivity $(p=0.138)$, however, the positivity was significantly higher in the age group of $\geq 45$ years $(p=0.039)$ than the other age groups (Table 2).

\section{DISCUSSION}

Skin surface biopsy (SSB) was firstly described by Marks and Dawber in 1971 as a simple, non-invasive examination technique to detect various types of pathology within the stratum corneum, especially some micro-organisms which

Table 2: Distribution and prevalences of all the seborrhoeic dermatitis (SD) cases in terms of gender and age according to $D$ folliculorum positivity

\begin{tabular}{llccrcc}
\hline SD group & & \multicolumn{2}{c}{ Demodex-negative } & Demodex-positive & $p$-values \\
\hline \multirow{2}{*}{ Age range (years) } & $18-30$ & 50 & $57.47 \%$ & 5 & $38.46 \%$ & \\
& $31-44$ & 27 & $31.03 \%$ & 3 & $23.08 \%$ & \\
\multirow{3}{*}{ Gender } & $\geq 45$ & 10 & $11.49 \%$ & 5 & $38.46 \%$ & $\mathbf{0 . 0 3 9}$ \\
& Male & 39 & $44.83 \%$ & 3 & $23.08 \%$ & \\
& Female & 48 & $55.17 \%$ & 10 & $76.92 \%$ & 0.138 \\
\hline
\end{tabular}

The distribution of the mite-positive patients with RSC according to the clinical types was five erythematelangiectatic and 33 papulopustular. In one patient with phymatous RSC, no mite was detected. The mean mite density in the papulopustular and erythematelangiectatic types was 8.66 and 9.6, respectively. Although most of the mite-positive RSC patients had the papulopustular type, there was no statistically significant difference according to the different clinical types $[p=0.168]$ (Table 3 ).

Table 3: Distribution of the patients with rosacea (RSC) according to the different clinical types of the disease

\begin{tabular}{lrcrrr}
\hline Clinical types of RSC & \multicolumn{2}{c}{ Mite-negative } & Mite-positive & $\boldsymbol{p}$-value \\
\hline Erythematelangiectatic & 17 & $27.42 \%$ & 5 & $8.06 \%$ & \\
Papulopustular & 44 & $70.97 \%$ & 33 & $53.23 \%$ & \\
Phymatous & 1 & $1.61 \%$ & 0 & $0.00 \%$ & 0.168 \\
\hline
\end{tabular}

In total, 51 out of the 200 patients were mite-positive. Prevalence of the mite-positive patients for the RSC group was $74.51 \%$ (38 patients: 28 women, 10 men) and $25.49 \%$ (13 patients: 10 women, three men) in the SD group. The difference between the two disease groups was statistically significant $[p=0.0001]$ (Table 4).

Table 4: Distribution of the patients with rosacea (RSC) and seborrhoeic dermatitis (SD) cases according to $D$ folliculorum positivity

\begin{tabular}{llcc}
\hline Diseases & Mite positivity & Mite negativity & Total \\
\hline Rosacea & $\mathbf{3 8}(74.51 \%)$ & $62(41.61 \%)$ & $\mathbf{1 0 0}$ \\
Seborrhoeic dermatitis & $\mathbf{1 3}(25.49 \%)$ & $87(58.39 \%)$ & $\mathbf{1 0 0}$ \\
\hline Total & $\mathbf{5 1}$ & $\mathbf{1 4 9}$ & $\mathbf{2 0 0}$ \\
\hline
\end{tabular}

$p=0.0001$ colonize the skin $(15,16)$. In 1993, Forton and Seys redefined and renamed this technique standardized skin surface biopsy $[\mathrm{SSSB}](15,17)$ and indicated that it provides a diagnostic tool with high specificity $(98 \%)$, with little false positivity [2\%] (17). Two species of Demodex mites are known to inhabit the human skin. The smaller one, $D$ brevis, is about $0.15-0.2 \mathrm{~mm}$ long and spindle-shaped, has four pairs of short legs and is located in the deep level of the skin, especially in the sebaceous or meibomian glands. The second is $D$ folliculorum, which is about $0.3-0.4 \mathrm{~mm}$ long, has an elongated-shape, four pairs of legs attached to the head-neck and is located in the hair follicles $(1,2)$. Both of the mites reside in sebaceous gland-rich areas $(1,2,9,11)$. Healthy individuals have $D$ folliculorum at rates as high as $20-30 \%$ $(6,18)$; density on healthy skin is normally $<5 \mathrm{~cm}^{2}$ with SSSB (6), with a mean density of $0.7(13,17)$. The mite can be transmitted to newborns a few days after birth through close physical contact; however, its density is normally low in childhood $(1,2,6)$. The prevalance increases with age due to the increase in sebum production $(6,11,19)$ and may reach $100 \%$ in the elderly $(2,6)$. The mean prevalence in men is more than in women $[23 \%$ vs $13 \%](9,12)$. Contrarily, Bonnar et al reported that there was no gender difference in prevalence (20). In our study, most mite-positive cases were women in each disease group [73.68\% in $\mathrm{RSC}, 76.92 \%$ in $\mathrm{SD}$, but statistically there was no difference according to the gender in either group. Additionally, the majority of the mite-positive RSC cases were in the age range of 45 years and over [52.63\%], but we did not find statistical difference between the age groups. Although the majority of our SD patients were in the younger population, the mite positivity was also higher in the age group of 45 years and over than the other age groups, and the result was significant $(p=0.039)$.

Our findings were compatible with the literature in that the increased sebaceous activation with age becomes a 
suitable medium for the growth of parasites. However, Ni Raghallaigh et al indicated that for the development of the mites, the nature of the sebum might be more important than its redundancy (21). Indeed, Zhao et al showed that a few patients with excessive Demodex had no obvious clinical symptoms (7). Moreover, Akilov and Mumcuoglu have provided evidence that people with $\mathrm{Cw} 2$ and $\mathrm{Cw} 4$ haplotypes are more susceptible to Demodex infestation $(14,22)$. In the pathogenesis, various mechanisms have been suggested: (i) an epidermal hyperplasia, reactive hyperkeratinization and secretory blockage as a result of the obstruction of the sebaceous canals and follicles with increasing bacterial colonization; (ii) stimulation of humoral/cellular immunity by the mite itself, its discharge products, or foreign-body reaction according to its chitin structures $(2,6,11,19,23-25)$ and (iii) autoinflammation triggered by a reaction between host and some Bacillus oleronius antigens of mites, because of resemblance to heat-shock proteins $(2,24,26)$.

Rosacea is one of the diseases located in sebaceous gland-rich areas. Although the roles of vascular and immunological alterations, reactive oxygen species, chemical and ingested agents, influence of the weather, dermal matrix degeneration, anomalies of the pilosebaceous unit and bacterial and parasitic agents have been suggested, the pathophysiology of RSC is not completely understood (27). In recent years, besides the aforementioned reasons, the role of the mite has increasingly been questioned in the pathogenesis of $\operatorname{RSC}(2,15,27,28)$ and it has been confirmed as one of the risk factors (29). Rosacea is three times more common in middle-aged women than in men (2, 5). Forton et al stated that the number of female patients was 2.5 times higher than males (13). Yücel and Yilmaz indicated that $67.85 \%$ of their patients with RSC were women, and mite positivity was $35.71 \%$ in females and $17.85 \%$ in males (30). However, Bonnar et al (31), Zhao et al (7) and Aycan et al (32) indicated that there was no difference according to gender. In our study, the count of female patients with RSC (72/100) was higher than males $(28 / 100)$, and the prevalences of RSC in the 18-30 and 31-44-year age ranges for women were significantly higher than the men of their respective age groups. However, we did not find statistically significant differences in the RSC patients in terms of gender and age according to $D$ folliculorum positivity.

Forton and Seys reported a mean mite density of $10.8 / \mathrm{cm}^{2}$ in 49 patients with RSC and a density of $0.7 / \mathrm{cm}^{2}$ in 45 control patients $(2,9,17)$. In another case-control study, Bonnar et al indicated the mean mite count, determined by $\mathrm{SSSB}$, as 49.8 in the RSC group and 10.8 in the control group (31). In the polymerase chain reaction (PCR)-based quantification study of Casas et al, D folliculorum was detected more frequently in RSC patients than age-matched healthy controls and the mean density was indicated as 5.7 times higher in RSC patients than in healthy persons (33). Yücel and Yilmaz (30) [with skin scrapings], and Aycan et al (32) [with SSSB] showed that in their patients with RSC, the prevalences of mite positivity were $60.7 \%$ and $61.5 \%$, respectively, but they did not mention mean mite density. In our study, prevalence of $D$ folliculorum in the RSC patients was much higher (74.51\%) than healthy persons (20-30\%), and was consistent with the literature. Although we obtained a lower mean mite density than the literature at 8.78 , this count was still 12.54 times higher than in healthy skin $(0.7)$. Considering all types of RSC, statistically higher mite density is found in cases with papulopustular $\operatorname{RSC}(17,33)$. In two different studies by Forton et al, the prevalences in papulopustular RSC were $74 \%$ and $55 \%$, respectively (13, 17) and the mean mite density was reported as 36 (13). In our study, we obtained much more positivity in the papulopustular type of RSC (53.23\%) than the other types, but there was no statistically significant difference. Although mean mite density in the papulopustular types (8.66) was lower than the erythematelangiectatic type (9.6), we thought this could be due to the limited number of patients with the second type.

Another disease that is located in sebaceous gland-rich areas is $\mathrm{SD}(6,14,34,35)$. In the aetiopathogenesis of $\mathrm{SD}$, many causes, including increased sebum activity, androgenassociated hormonal factors, immunological abnormalities, genetic predisposition [HLA-AW30, AW31 and B12], emotional stress, neurological disorders (35), fungal infections, nutritional deficits (34), lifestyle, environmental factors, pitrosporum ovale infection, drugs and $D$ folliculorum $(6,36,37)$, have been suggested. The currently accepted theory on the pathogenesis suggests genetic predisposition to Malessezia species which produce unsaturated fatty acids leading to a non-immunogenic irritation (35). Contrary to RSC, the association between SD and Demodex mites has not yet been confirmed. However, it has been suggested that the $D$ folliculorum mite might cause SD with similar mechanisms as those of Malessezia $(6,38)$. Karincaoglu et al stated that reactivation of the immune system by $D$ folliculorum antigens or its toxic products could stimulate keratinocyte-originated cytokines, which in turn might cause SD inflammation, or aggravate SD. They also stated that in both lesional and non-lesional areas of patients with SD, the number of Demodex mites was significantly higher $(50 \%)$ than healthy controls $(13.1 \%)$, and the mean density was 8.6 and 1.03 in the patients and the controls, respectively (6). In our study, mite positivity detected in the patients with SD was a low rate of $13 \%$, which was similar to that of healthy persons. The mean mite density of our SD patients was 11.15, and this count was higher than in both healthy individuals and the results of Karincaoglu et al. Seborrhoeic dermatitis has two incidence peaks: the first in newborn infants up to three months old, and the other in adults around 30-60 years old. Men are affected more often than women $(34,35)$. Karincaoğlu et al also stated that the mean age of patients was 36.71 years, and the majority of them were men $[30 / 38 ; 78.9 \%]$ (6). In our study, the mean age of the patients with SD was 33.04 years [mean age of 
mite-positive SD patients was 38.15 years] and the majority of the patients were in the 18-30-year age range $(55 / 100)$; these findings were consistent with the literature. In contrast to the literature, the majority of our patients with SD were women $(58 / 100 ; 58 \%)$. Additionally, the mite positivity was significantly higher in the age group of 45 years and over than the other age groups ( $p=0.039)$; however, there was no statistical significance in terms of gender. Our findings supported the literature that the increased sebaceous activation with age prepares a suitable medium for growth of Demodex mites. Because the majority of the patients were women and inconsistent with the literature, we think that gender factor alone is not enough for the increase in mite density in SD.

Finally, when we compared the prevalence of the mites in both diseases, there was a significant difference. The prevalence of the mite-positive patients with RSC (74.51\%) was approximately three times higher than the patients with SD (25.49\%). Therefore, we think that $D$ folliculorum may play a more important role in the pathogenesis of RSC than SD. Additionally, even if the prevalence of $D$ folliculorum in the patients with SD was not different from healthy persons, we found a much higher mean mite count compared to the healthy population. Therefore, we believe that the $D$ folliculorum mite may play a role in the pathogenesis of SD, at least in some ways. Moreover, due to higher mean mite counts in the SD and erythematotelangiectatic RSC than in papulopustular RSC, we think that the aetiopathological role of the $D$ folliculorum in both diseases may depend on not only the number of the mites and their follicular inflammatory effects, but also, and perhaps more importantly, their immunogenetic pathogenicity or non-immunogenic irritative effects. Although our results suggest that the $D$ folliculorum mite may play a more important role in the aetiopathogenesis of RSC than SD, further comparative studies are needed to support the obtained results.

\section{CONCLUSION}

In conclusion, the following results were obtained in our study: (i) there were no significant differences in $D$ folliculorum positivity in the patients with RSC in terms of gender and age, and mean mite count in RSC was 8.78; (ii) in the SD group, there was no statistical significance in terms of gender according to mite positivity, however, the positivity was significantly higher in the age group of 45 years and over than the other age groups. The mean mite count in this group was 11.15; (iii) in mite-positive RSC patients, there was no significant difference with respect to the different clinical types of RSC and (iv) the $D$ folliculorum prevalence of the patients with RSC was approximately three times higher than the patients with $\mathrm{SD}$.

\section{REFERENCES}

1. Buechner SA. Rosacea: an update. Dermatology 2005; 210: 100-8.

2. Jarmuda S, O'Reilly N, Zaba R, Jakubowicz O, Szkaradkiewicz A, Kavanagh K. Potential role of Demodex mites and bacteria in the induction of rosacea. J Med Microbiol 2012; 61: 1504-10.

3. Powell FC. Clinical practice: rosacea. N Engl J Med 2005; 352: 793 803.

4. Wilkin J, Dahl M, Detmar M, Drake L, Feinsrein A, Odom R et al. Standard classification of rosacea: report of the National Rosacea Society Expert Committee on the classification and staging of rosacea. J Am Acad Dermatol 2002; 46: 584-7.

5. Butterwick KJ, Butterwick LS, Han A. Laser and light therapies for acne rosacea. J Drugs Dermatol 2006; 5: 35-9.

6. Karincaoglu Y, Tepe B, Kalayci B, Atambay M, Seyhan M. Is Demodex folliculorum an aetiological factor in seborrhoeic dermatitis? Clin Exp Dermatol 2009; 34: 516-20.

7. Zhao YE, Peng Y, Wang XL, Wu LP, Wang M, Yan HL, Xiao SX. Facial dermatosis associated with Demodex: a case-control study. J Zhejiang Univ Sci B 2011; 12: 1008-15.

8. Desch C, Nutting WB. Demodex folliculorum (Simon) and D brevis Akbulatova of man: redescription and reevaluation. J Parasitol 1972; 58: $169-77$

9. Bikowski JB, Del Rosso JQ. Demodex dermatitis: a retrospective analysis of clinical diagnosis and successful treatment with topical crotamiton. J Clin Aesthet Dermatol 2009; 2: 20-5.

10. Holzchuh FG, Hida RY, Moscovici BK, Villa Albers MB, Santo RM, Kara-Jose $\mathrm{M}$ et al. Clinical treatment of ocular Demodex folliculorum by systemic ivermectin. Am J Ophtalmol 2011; 151: 1030-4.

11. Basta-Juzbasic A, Subic JS, Ljubojevic S. Demodex folliculorum in development of dermatitis rosaceiformis steroidica and rosacea-related diseases. Clin Dermatol 2002; 20: 135-40.

12. Aylesworth R, Vance C. Demodex folliculorum and Demodex brevis in cutaneous biopsies. J Am Acad Dermatol 1982; 7: 583-9.

13. Forton F, Germaux MA, Brasseur T, De Liever A, Laporte M, Mathys $\mathrm{C}$ et al. Demodicosis and rosacea: epidemiology and significance in daily dermatologic practise. J Am Acad Dermatol 2005; 52: 74-87.

14. Karincaoglu Y, Bayram N, Aycan O, Esrefoglu M. The clinical importance of Demodex folliculorum presenting with nonspecific facial signs and symptoms. J Dermatol 2004; 31: 618-26.

15. Aşkin D, Seçkin D. Comparison of the two techniques for measurement of the density of Demodex folliculorum: standardized skin surface biopsy and direct microscopic examination. Br J Dermatol 2010; 162: 1124-6.

16. Marks R, Dawber RP. Skin surface biopsy: an improved technique for the examination of the horny layer. Br J Dermatol 1971; 84: 117-23.

17. Forton F, Seys B. Density of Demodex folliculorum in rosacea: a casecontrol study using standardized skin-surfaced biopsy. Br J Dermatol 1993; 128: 650-9.

18. Purcell SM, Hayes TJ, Dixon SL. Pustular folliculitis associated with Demodex folliculorum. J Am Acad Dermatol 1986; 15: 1159-62.

19. Roihu T, Karinieni A-L. Demodex mites in acne rosacea. J Cutan Pathol 1998; 25: 550-2.

20. Bonnar E, Eustace P, Powell FC. Demodex mite in normal skin. Lancet 1991; 337: 1168 .

21. Ni Raghallaigh S, Bender K, Lacey N, Brennan L, Powell FC. The fatty acid profile of the skin surface lipid layer in papulopustular rosacea. $\mathrm{Br}$ J Dermatol 2012; 166: 279-87.

22. Akilov OE, Mumcuoglu KY. Association between human demodicosis and HLA class I. Clin Exp 2013; 28: 70-3.

23. Morras PG, Santos SP, Imedio IL, Echeverria ML, Hermosa JM. Rosacea-like demodicidosis in an immmunocompromised child. Pediatr Dermatol 2003; 20: 28-30.

24. Lacey N, Delaney S, Kavanagh K, Powell FC. Mite related bacterial bacterial antigens stimulate inflammatory cells in rosacea. $\mathrm{Br} \mathrm{J}$ Dermatol 2007; 157: 474-81. 
25. Baima B, Sticherling M. Demodicidosis revisited. Acta Derm Venereol 2002; 82: 3-6.

26. Li J, O’Reilly N, Sheha H, Katz R, Raju VK, Kavanagh K et al. Correlation between ocular Demodex infestation and serum immunorectivity to Bacillus proteins in patients with facial rosacea. Ophthalmology 2010; 117: 870-7.

27. Rios-Yuil JM, Mercadillo-Perez P. Evaluation of Demodex folliculorum as a risk factor for the diagnosis of rosacea in skin biopsies. Mexico's General Hospital (1975-2010). Indian J Dermatol 2013; 58: 157.

28. O'Reilly N, Bergin D, Reeves EP, McElvaney NG, Kavanagh K. Demodex-associated bacterial proteins induce neutrophil activation. $\mathrm{Br}$ J Dermatol 2012; 166: 753-60.

29. Zhao YE, Wu LP, Peng Y, Cheng H. Retrospective analysis of the association between Demodex infestation and rosacea. Arch Dermatol 2010; 146: 896-902

30. Yücel A, Yilmaz M. Investigation of the prevalance of Demodex folliculorum and Demodex brevis in rosacea patients. Türkiye Parazitol Derg 2013; 37: 195-8.

31. Bonnar E, Eustace P, Powell FC. The Demodex mite population in rosacea. J Am Acad Dermatol 1993; 28: 443-8.

32. Aycan OM, Otlu GH, Karaman U, Daldal N, Atambay M. Frequency of the appearance of Demodex sp. in various patient and age groups. Türkiye Parazitol Derg 2007; 31: 115-8.

33. Casas C, Paul C, Lahfa M, Livideanu B, Lejeune U, Alverez-Georges S et al. Quantification of Demodex folliculorum by PCR in rosacea and its relationship to skin innate immune activation. Exp Dermatol 2012; 21: $906-10$.
34. Schwartz RA, Janusz CA, Janniger CK. Seborrheic dermatitis: an overview. Am Fam Physician 2006; 74: 125-30.

35. Sampaio AL, Mameri AC, Vargas TJ, Ramos-e-Silva M, Nunes AP, Carneiro SC. Seborrheic dermatitis. An Bras Dermatol 2011; 86: 1061 71.

36. Gupta AK, Bluhm R. Seborrheic dermatitis. J Eur Acad Dermatol Venereol 2004; 18: 13-26.

37. Valia RG. Etiopathogenesis of seborrheic dermatitis. Indian J Dermatol Venereol Leprol 2006; 72: 253-5.

38. Forton F, Song M. Limitations of standardized skin surface biopsy in measurement of the density of Demodex folliculorum. A case report. $\mathrm{Br}$ J Dermatol 1998; 139: 697-700.

Received 24 Feb 2015

Accepted 30 Mar 2015

Published 30 Sep 2015

Online: http://www.mona.uwi.edu/wimjopen/article/1650

(C) Tas et al 2015

This is an open access article made freely available under Creative Commons Attribution 4.0 International (CC BY 4.0). Users are free to share, copy and adapt this work as long as the copyright holder (author) is appropriately and correctly credited. See http://creativecommons. org/licences/by/4.0/deed.en_us for more information. 